\section{(2) OPEN ACCESS}

\title{
Gastric microbes associated with gastric inflammation, atrophy and intestinal metaplasia 1 year after Helicobacter pylori eradication
}

\author{
Joseph J Y Sung, Olabisi Oluwabukola Coker, Eagle Chu, Chun Ho Szeto, \\ Simson Tsz Yat Luk, Harry Cheuk Hay Lau, Jun Yu
}

Additional material is published online only. To view please visit the journal online (http://dx.doi.org/10.1136/ gutjnl-2019-319826).

Correspondence to Professor Joseph J Y Sung, Department of Medicine and Therapeutics, Chinese University of Hong Kong, New Territories, Hong Kong:

jjysung@cuhk.edu.hk

Received 9 September 2019 Revised 3 December 2019 Accepted 14 December 2019 Published Online First 23 January 2020
Check for updates

(C) Author(s) (or their employer(s)) 2020. Re-use permitted under CC BY-NC. No commercial re-use. See rights and permissions. Published by BMJ.

To cite: Sung JJY, Coker 00, Chu E, et al. Gut

2020:69:1572-1581.

\begin{abstract}
Objective Helicobacter pylori is associated with gastric inflammation, precancerous gastric atrophy (GA) and intestinal metaplasia (IM). We aimed to identify microbes that are associated with progressive inflammation, GA and IM 1 year after $H$. pylori eradication.
\end{abstract}

Design A total of $587 \mathrm{H}$. pylori-positive patients were randomised to receive $H$. pylori eradication therapy (295 patients) or placebo (292 patients). Bacterial taxonomy was analysed on 404 gastric biopsy samples comprising 102 pairs before and after 1 year $H$. pylori eradication and 100 pairs before and after 1 year placebo by 165 rRNA sequencing.

Results Analysis of microbial sequences confirmed the eradication of $H$. pylori in treated group after 1 year. Principal component analysis revealed distinct microbial clusters reflected by increase in bacterial diversity $(p<0.00001)$ after H. pylori eradication. While microbial interactions remained largely unchanged after placebo treatment, microbial co-occurrence was less in treated group. Acinetobacter Iwoffii, Streptococcus anginosus and Ralstonia were enriched while Roseburia and Sphingomonas were depleted in patients with persistent inflammation 1 year after $H$. pylori eradication. A distinct cluster of oral bacteria comprising Peptostreptococcus, Streptococcus, Parvimonas, Prevotella, Rothia and Granulicatella were associated with emergence and persistence of GA and IM. Probiotic Faecalibacterium praustznii was depleted in subjects who developed GA following $H$. pylori eradication. Functional pathways including amino acid metabolism and inositol phosphate metabolism were enriched while folate biosynthesis and NOD-like receptor signalling decreased in atrophy/IMassociated gastric microbiota.

Conclusion This study identified that gastric microbes contribute to the progression of gastric carcinogenesis after $H$. pylori eradication.

\section{INTRODUCTION}

Independent epidemiological studies have confirmed that infection with Helicobacter pylori is the most important acquired aetiological agent for gastric cancer, ${ }^{1}$ a global leading cause of cancerrelated deaths. The chronic inflammation induced by $H$. pylori induces several histopathological changes in the gastric epithelium and maintains a constant production of a cascade of cytokines which in turn attracts immune cells that generate oxidative

\section{Significance of this study}

What is already known on this subject?

- Helicobacter pylori infection is associated with gastric inflammation, precancerous gastric atrophy and intestinal metaplasia.

- Less than $3 \%$ of $H$. pylori-infected individuals develop gastric cancer, and about $20 \%$ of individuals with chronic gastritis are $\mathrm{H}$. pylori negative; other organisms may induce gastric inflammation and gastric carcinogenesis.

What are the new findings?

- Microbial co-occurrence was reduced and characterised by a distinct cluster of oral bacteria 1 year after eradication of $H$. pylori, while gastric microbial ecology remained largely unchanged 1 year after placebo treatment.

- One year after H. pylori eradication, Acinetobacter Iwoffii, Streptococcus anginosus and Ralstonia were enriched while Roseburia and Sphingomonas were depleted in patients with persistent inflammation.

- A distinct cluster of oral bacteria comprising Peptostreptococcus, Streptococcus, Parvimonas, Prevotella, Rothia and Granulicatella were associated with emergence and persistence of atrophy and intestinal metaplasia, while probiotic Faecalibacterium praustznii was depleted in subjects who developed atrophy 1 year after $H$. pylori eradication.

- Amino acid metabolism and inositol phosphate metabolism were enriched while folate biosynthesis and NOD-like receptor signalling decreased in subjects with emerged intestinal metaplasia following $H$. pylori eradication.

How might it impact on clinical practice in the foreseeable future?

- This study demonstrated the contribution of gastric microbes in the development and perpetuation of precancerous gastric lesions after $H$. pylori eradication.

- The identified microbes that associated with progression of gastric inflammation, atrophy or intestinal metaplasia are potential therapeutic targets in gastric cancer prevention. 
radicals with the potential to damage host DNA. ${ }^{2}$ The mechanism employed by $H$. pylori in promoting the emergence of pre-neoplastic gastric lesions (atrophy and intestinal metaplasia) is predominantly chronic inflammation. Treatment regimens targeted at $\mathrm{H}$. pylori eradication have been demonstrated to be effective in preventing the progression of pathological changes in the gastric mucosa. ${ }^{3}$ However, despite the reported effectiveness of eradication therapy, some patients do continue to develop pre-neoplastic gastric lesions including gastric atrophy (GA) and intestinal metaplasia (IM). ${ }^{3}$ Notably, less than $3 \%$ of H. pylori-infected individuals develop gastric cancer, ${ }^{5}$ and about $20 \%$ of individuals with chronic gastritis are $H$. pylori negative, suggesting that other organisms may induce gastric inflammation and even gastric carcinogenesis. ${ }^{6}$

It is possible that, while $H$. pylori initiate the inflammatory process in the stomach, other gastric microbes with proinflammatory potential play an important role in maintaining the progression of inflammation and dysplastic changes leading to development of gastric cancer. The loss of acid-secreting parietal cells induced by chronic $H$. pylori infection in the gastric epithelium may provide a favourable environment for colonisation by non- $H$. pylori microbes to induce further pro-carcinogenic process. Moreover, the 'point of no return' in the cascade of events leading to gastric cancer is reportedly associated with patients with IM and dysplasia, independent of $H$. pylori status. ${ }^{2}$

The relevance of non-H. pylori gastric microbes in the development of gastric cancer was previously demonstrated by using transgenic INS-GAS mice, overexpressing gastrin. Antibiotictreated INS-GAS mice colonised with $H$. pylori had delayed onset of gastric cancer compared with control mice infected with $H$. pylori without antibiotic treatment, ${ }^{7}$ suggesting that gastric microbes could enhance the effects of $H$. pylori in gastric carcinogenesis. Single-nucleotide polymorphisms in host genes that influence bacterial pattern recognition receptors in gastric cancer patients have also been reported, ${ }^{8}$ underscoring the active role of bacteria-host cross-talk in gastric tumourigenesis. It is therefore pertinent to investigate bacteria that are associated with inflammation, atrophy and IM after the eradication of H. pylori to establish a surveillance programme based on gastric cancer risk stratification specific to non- $H$. pylori-infected patients

Here, we carried out a retrospective study involving a 1-year follow-up of $H$. pylori-positive subjects who received $H$. pylori eradication therapy compared with those who received placebo. We identified gastric microbes that are associated with gastric inflammation, atrophy and IM after $\mathrm{H}$. pylori eradication.

\section{MATERIALS AND METHODS \\ Patients}

A total of $587 \mathrm{H}$. pylori-positive patients residing in Yantai county of Shandong Province, China, with incidence of 50 gastric cancer cases per 100000 population, were randomised as described before, ${ }^{4}$ of which $295 \mathrm{H}$. pylori-positive patients received a 1-week course of omeprazole $(20 \mathrm{mg})$, amoxicillin $(1 \mathrm{~g})$ and clarithromycin $(500 \mathrm{mg})(\mathrm{OAC}$ treatment) twice daily, while $292 \mathrm{H}$. pylori-positive subjects received placebo (placebo treatment). Subjects underwent endoscopy with biopsy with sterile forceps at baseline and after 1 year from the same sites of both the antrum and corpus. Histology and urease test were used to confirm the eradication of $H$. pylori. Tissue biopsies were also obtained during endoscopy and frozen immediately at $-80^{\circ} \mathrm{C}$.

\section{Histological assessment}

Severity of gastric inflammation, GA and IM was graded according to the updated Sydney classification by a pathologist who was unaware of the treatment condition. Progression and regression were defined as increase or decrease, respectively, of inflammation, GA or IM scores after 1 year.

\section{Sample sequencing}

Purification of DNA for $16 \mathrm{~S}$ rRNA gene sequencing was performed on a total of 404 gastric biopsy samples, comprising 100 pairs before and after 1 year of placebo treatment, and 102 pairs before and after eradication of $H$. pylori by OAC treatment (online supplementary table 1). Gastric biopsies tissues were digested using lysozyme and mutanolysin enzymes (Sigma, Hong Kong), followed by bead beating and DNA purification with QIAamp DNA Mini Kit. Illumina Primer pair 515 f, 5'-GTGCCAGCMGCCGCGGTAA-3' and 806r, 5'-GGACTACHVGGGTWTCTAAT-3' targeted across the V4 hypervariable regions of the 16S rRNA gene was used for sequencing on Illumina MiSeq platform. Invitrogen SequelPrep Normalization Plate Kit was used for Library clean-up and normalisation.

\section{Quality control and annotation of sequences}

Mothur software ${ }^{9}$ suite was used for sequence curation and analysis. Briefly, Needleman-Wunsch alignment algorithm was used to merge paired-end reads into contigs with default parameters. This was followed by alignment against SILVA $16 \mathrm{~S}$ rRNA sequence database (V.123) with nearest alignment space termination (NAST) algorithm. ${ }^{10}$ Contigs that mapped within V4 region were trimmed and merged with sequences having a difference of most two nucleotide bases, followed by chimeric sequences screening using de novo Uchime. ${ }^{11}$ Greengenes database (V.13.8) was used for taxonomy assignment. Reads assigned as nonbacterial or unknown kingdoms were discarded. Average neighbour clustering algorithm was the used to cluster the resulting sequences into operational taxonomic units (OTUs), with 97\% identity cut-off. The functional potential of the gastric microbial communities was estimated by the PICRUSt ${ }^{12}$ algorithm. Kyoto Encyclopedia of Genes and Genomes (KEGG) database was used for pathway enrichment analysis.

\section{Statistical analyses}

Differentially abundant non- $H$. pylori microbes and predicted microbiota functional alterations associated with inflammation, GA and IM were analysed using Lefse algorithm. ${ }^{13}$ Differences with $\log 10$ linear discriminant analysis scores (absolute values) $>2.0$ and $\mathrm{p}$ value $<0.05$ were considered significant. Co-occurrence and co-exclusion relationships between bacteria with relative abundance greater than $0.1 \%$, and with Helicobacter at the genus level were estimated using SparCC algorithm, ${ }^{14}$ known for its robustness to gut microbiome data compositionality. $\mathrm{P}$ values less than 0.05 after multiple comparisons correction using false discovery rate method were considered significant. Cytoscape V.3.0.4 was used for visualisation of bacteria co-occurrence. Network parameters including topological coefficient, closeness centrality and stress were estimated using the network analyser algorithm of Cytoscape V.3.0.4 with default parameters, and compared using Mann-Whitney U test.

\section{RESULTS}

\section{Eradication of $H$. pylori with OAC treatment}

Acute and chronic gastritis decreased in both antrum and corpus of the stomach in patients after $H$. pylori eradication. In the 


\begin{tabular}{lcl}
\hline Table 1 Demographic of study subjects & \\
\hline Number of subjects & $\begin{array}{l}\text { OAC-treated group } \\
(\mathbf{n}=102)\end{array}$ & $\begin{array}{l}\text { Placebo group } \\
(\mathbf{n}=100)\end{array}$ \\
\hline Inflammation at baseline & 102 & 100 \\
\hline Inflammation at after 1 year & 16 & 91 \\
\hline Atrophy at baseline & 17 & 15 \\
\hline Atrophy after 1 year & 37 & 40 \\
\hline Intestinal metaplasia at baseline & 49 & 45 \\
\hline Intestinal metaplasia after 1 year & 24 & 29 \\
\hline
\end{tabular}

OAC, omeprazole, amoxicillin and clarithromycin.

placebo group, antral biopsy specimens had more pronounced acute gastritis and a marginal increase in atrophy compared with OAC-treated group. Changes in IM were, however, similar between placebo and OAC-treated group. ${ }^{3}$

Analysis of $16 \mathrm{~S}$ rRNA gene sequences was performed on a total of 404 gastric biopsy samples, comprising 100 pairs before and after 1 year of placebo treatment, and 102 pairs before and 1 year after OAC treatment. Demographic details of subjects are presented in table 1 . The eradication of $H$. pylori was confirmed in the OAC-treated group with relative abundance of $H$. pylori of $0.016 \pm 0.0018$ compared with pre-treatment $(0.68 \pm 0.027)$, pre-placebo $(0.67 \pm 0.025)$ and post-placebo $(0.46 \pm 0.023)$ $(\mathrm{p}<0.00001)$ groups (figure 1A). Principal component analysis revealed a distinct gastric microbiota cluster for OAC-treated group (figure 1B). Eradication of $H$. pylori was associated with increase in bacterial diversity $(\mathrm{p}<0.00001)$ (figure $1 \mathrm{C})$, reflected by proliferation of several bacterial species (figure 1D). However, with the exclusion of $H$. pylori, the increase in bacteria diversity was no longer observed, indicating that the increased diversity resulted from the dominant abundance of $H$. pylori before OAC therapy. The most abundant bacteria families after $H$. pylori eradication with relative abundance greater than $1 \%$ are Pseudomonadaceae, Oxalobacteriaceae, Microbacteriaceae, Enterobacteriaceae, Lachnospiraceae, Vibrionaceae and Halomonadaceae (online supplementary figure 1). They have increased respectively by $3.9,4.6,4.2,2.3,1.1,6.9$ and 5.7 folds 1 year after $H$. pylori eradication. In addition to Helicobacter, Haemophilus $(\mathrm{p}=0.0061)$, Actinobacillus $(\mathrm{p}=0.0019)$ and Neisseria $(p=0.0056)$ were observed to be reduced after OAC treatment (figure 1E) and were represented by Haemophilus influenzae Otu42, Actinobacillus parahaemolyticus_Otu100 and Neisseria subflava at OTU level (online supplementary table 2). Samples with $>1 \% H$. pylori relative abundance in the OAC-treated group were regarded as being positive for $\mathrm{H}$. pylori infection as previously described ${ }^{15}$ and were excluded from downstream analysis.

\section{Microbiota ecology is altered after $\boldsymbol{H}$. pylori eradication}

Gastric microbial co-occurrence remained largely unchanged in the placebo-treated group (figure $2 \mathrm{~A}, \mathrm{~B}$, online supplementary tables 3-4). Much less microbial co-occurrence was, however, observed after a year following the eradication of $H$. pylori by OAC treatment (figure 2C,D, online supplementary tables 5-6). Network topology analysis showed that topological coefficient $(p=0.023)$ closeness centrality $(p=0.016)$ and stress $(p=0.01)$ of the bacteria nodes were statistically different in the microbial ecological network in the absence of $H$. pylori compared with the $H$. pylori-infected placebo group. In addition, $H$. pylori had predominantly co-exclusive association with other gastric microbes in the $H$. pylori-infected groups. In the OAC-treated

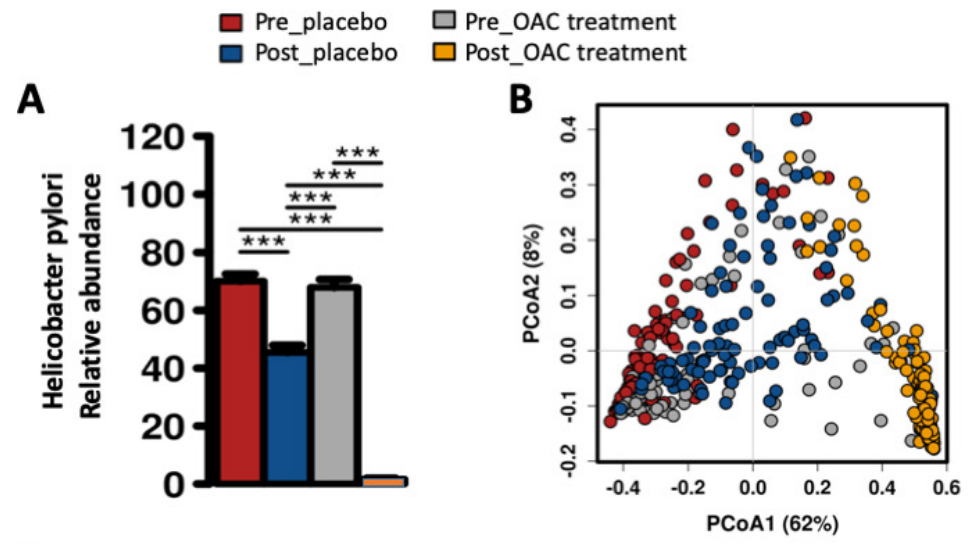

D

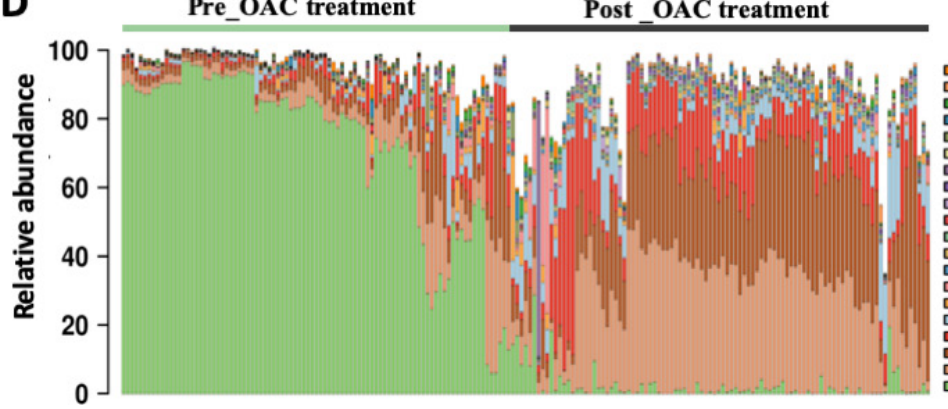

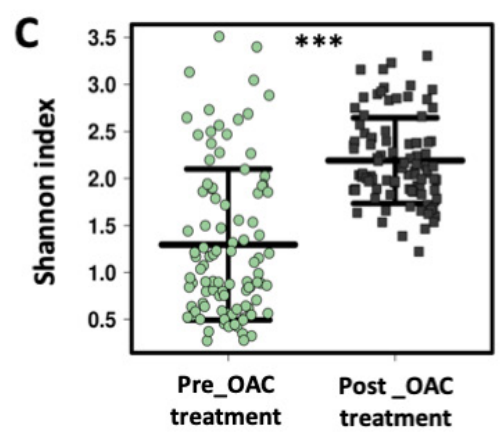

$\mathbf{E}$

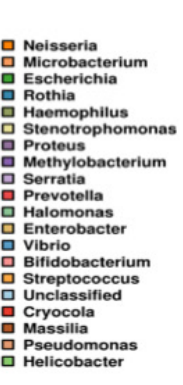

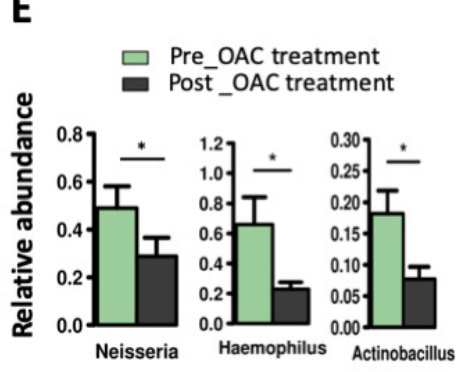

Figure 1 Eradication of Helicobacter pylori with omeprazole, amoxicillin and clarithromycin (OAC) treatment. (A) Relative abundance of $H$. pylori in Pre_placebo, Post_placebo, Pre_OAC treatment and Post_OAC treatment groups. (B) Principal component analysis plot showing a distinct cluster for Post_OAC treatment group. (C) Bacterial diversity estimated by Shannon index for Pre_OAC treatment and Post_OAC treatment groups. (D) Top 20 bacterial genera before and after 1 year of OAC treatment. (B) Relative abundance of Neisseria, Haemophilus and Actinobacillus 1 year after OAC treatment. ${ }^{*}$ Adjusted $p<0.05,{ }^{* *}$ adjusted $p<0.01,{ }^{* * *}$ adjusted $p<0.001$. 
A Pre_placebo

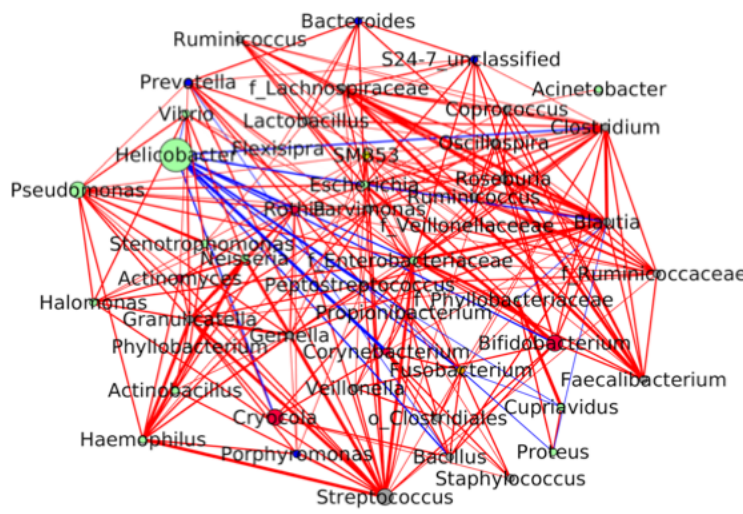

C Pre_OAC_treatment

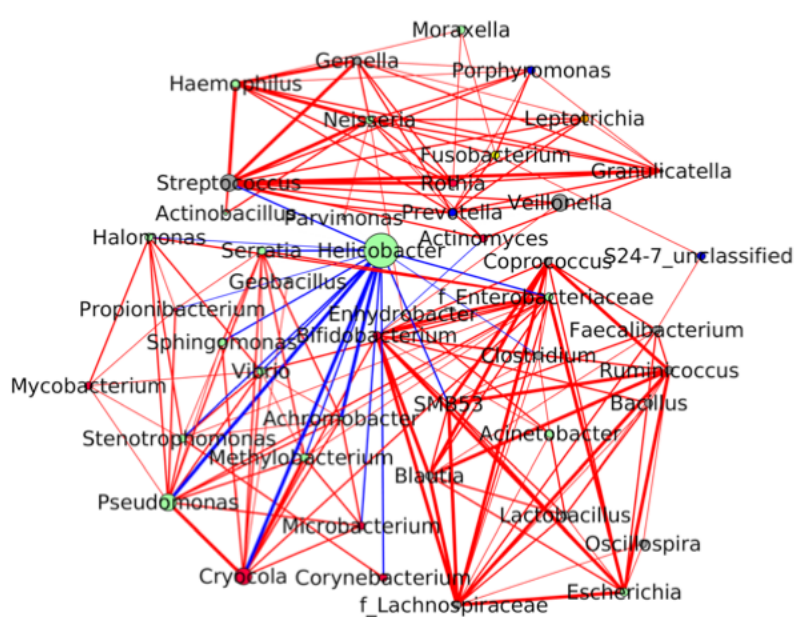

D

\section{B Post_placebo}
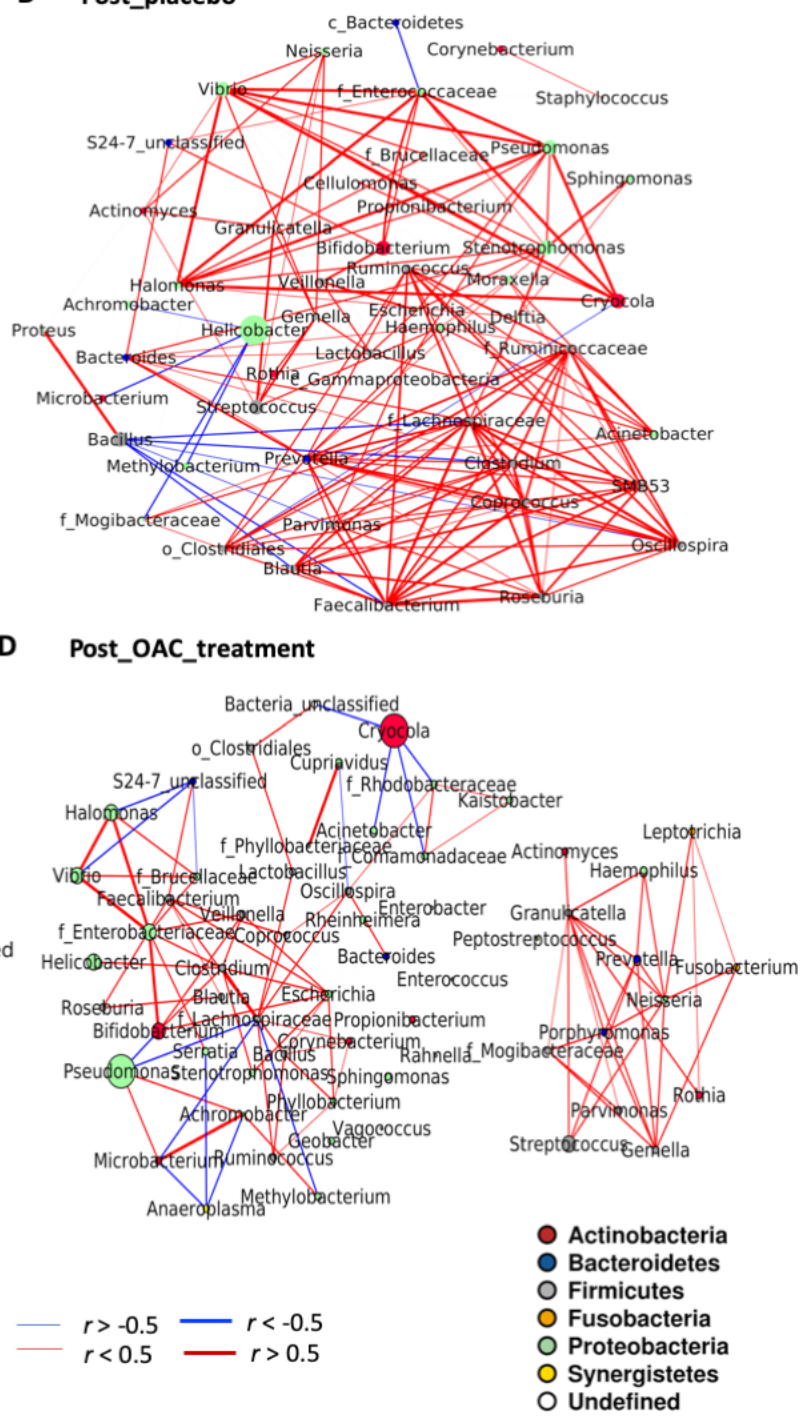

Figure 2 Microbiota ecology is altered after Helicobacter pylori eradication. Ecological co-occurrence network in (A) Pre_placebo, (B) Post_placebo, (C) Pre_OAC treatment and (D) Post_OAC treatment groups. Significant $(Q>0.05)$ SparCC algorithm was used to infer co-occurrence among top $(0.1 \%$ relative abundance) bacterial genera and with Helicobacter.

group, a distinct cluster of oral bacteria including Peptostreptococcus, Parvimonas, Fusobacterium, Haemophilus, Neisseria, Gemella, Granulicatella, Rothia, Streptococcus and Porphyromonas was observed (figure 2D). The change in gastric microbial ecology after $H$. pylori eradication indicates that $H$. pylori impacts the interactions of other gastric microbes which may contribute to inflammation and development of cancerous lesions in infected patients.

\section{Bacteria associated with inflammation after $H$. pylori eradication}

The intensities of polymorphonuclear infiltration in acute inflammation decreased in $84.1 \%$ of patients after $H$. pylori eradication. ${ }^{3}$ We then determined the bacteria that may potentially contribute to the persistent inflammation in patients after $H$. pylori eradication. Using linear discriminant analysis with effect size (Lefse), we identified Acinetobacter, Ralstonia, Actinobacillus and Erwinia as enriched while Sphingomonas and Roseburia as depleted bacterial genera in patients with inflammation in the absence of $H$. pylori (figure 3A). At the OTU level, Acinetobacter iwoffii_Otu180, Actinobacillus parahaemolyticus Otu100, Prevotella nanceiences Otu232, Erwinia soli_Otu664, Streptococcus anginosus_Otu27̄8, Acinetobacter rhizosphaerae_Otu208 and Acinetobacerguillouiae_Otu81 were enriched in patients with persistent inflammation 1 year after $H$. pylori eradication (figure $3 \mathrm{~B}$ ).

\section{Non-H. pylori bacteria associated with gastric atrophy}

Histological changes induced by chronic $H$. pylori infection may be aggravated by gastric microbes. We investigated differentially abundant bacteria associated with atrophy before and after OAC treatment. Our analysis revealed that Moraxella, Pasteurella, Bulleidia and Agrobacterium were enriched in patients with atrophy before $H$. pylori eradication therapy (figure 4A). After H. pylori eradication treatment, atrophy scores decreased in $20.8 \%$ of subjects. ${ }^{3}$ Correlation analysis on atrophy scores and bacteria abundance in OAC-treated group revealed that Granulicatella $(\mathrm{R}=0.41, \mathrm{p}=0.0027)$, Abiotrophia $(\mathrm{R}=0.31, \mathrm{p}=0.023)$ 


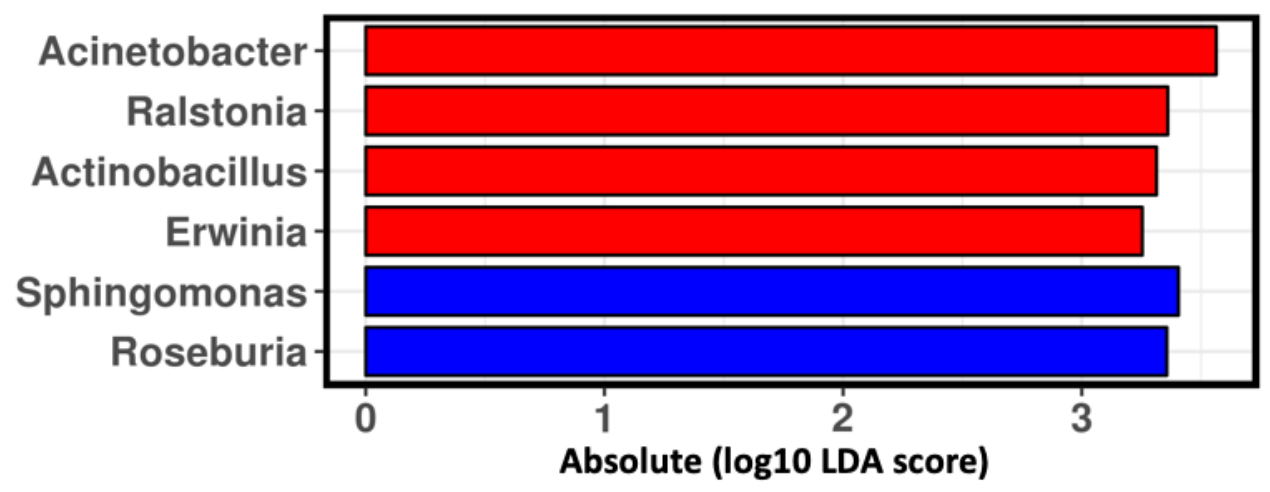

B

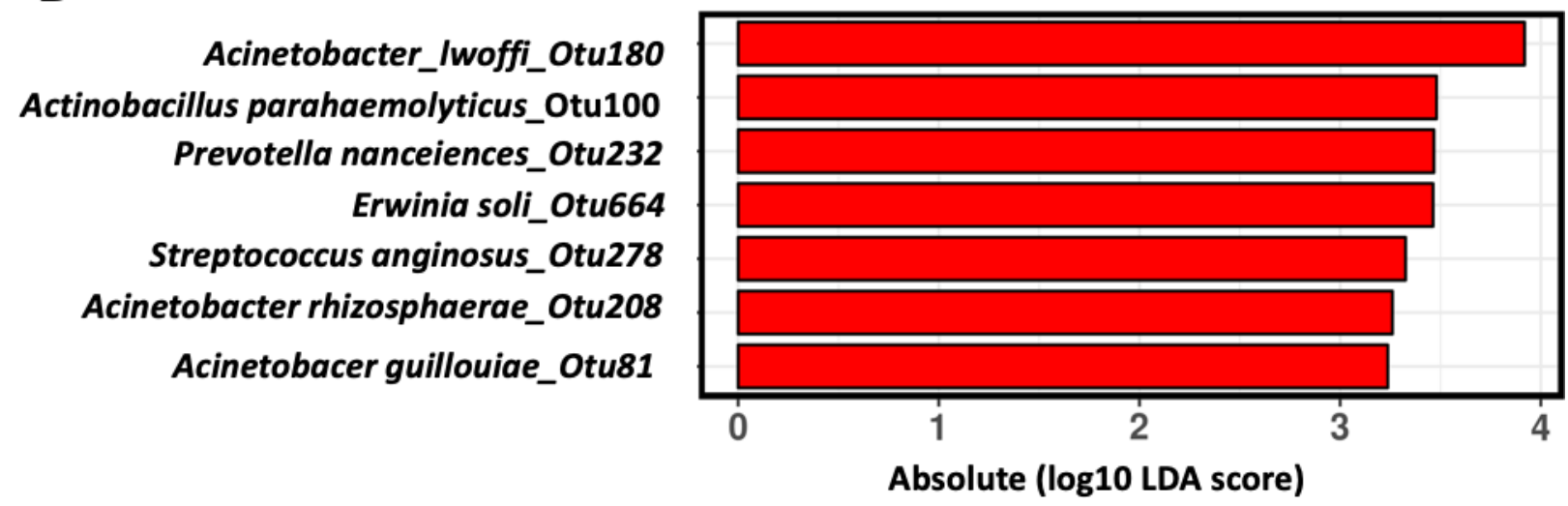

Figure 3 Bacteria associated with inflammation after Helicobacter pylori eradication. Linear discriminant analysis (LDA) with effect size (Lefse) showing (A) bacterial genera and (B) bacterial OTU associated with inflammation 1 year after $H$. pylori. OTU, operational taxonomic unit.

and Peptostreptococcus $(\mathrm{R}=0.31, \mathrm{p}=0.028)$ were positively correlated, while Faecalibacterium $(\mathrm{R}=-0.43, \mathrm{p}=0.0013)$, Rahnella ( $\mathrm{R}=-0.38, \mathrm{p}=0.005)$, Bacteroides $(\mathrm{R}=-0.34$, $\mathrm{p}=0.012)$, Clostridium $(\mathrm{R}=-0.29, \mathrm{p}=0.035)$ and Acinetobacter $(\mathrm{R}=-0.27, \mathrm{p}=0.046)$ were negatively correlated with atrophy scores (figure 4B). We further observed the enrichment of Granulicatella, Streptococcus, Rothia and Leptotrichia in subjects with emerged atrophy 1 year after $H$. pylori eradication (figure 4C). Acinetobacter, Faecalibacterium, Rahnella, Kaistobacter, Blautia, Caulobacter, Norcadioides and Brevundimonas were more abundant in subjects with no atrophy development following $H$. pylori eradication.
A

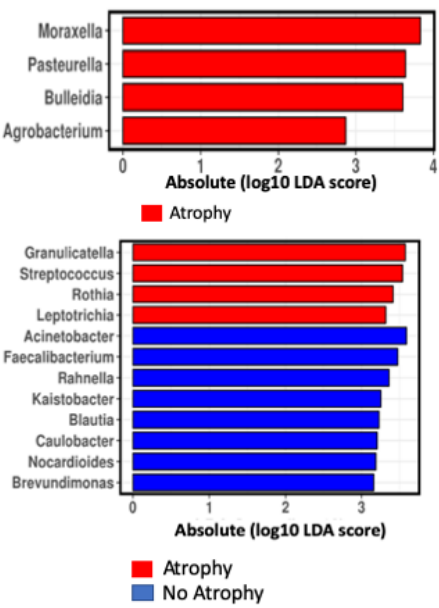

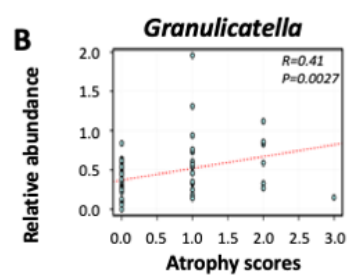
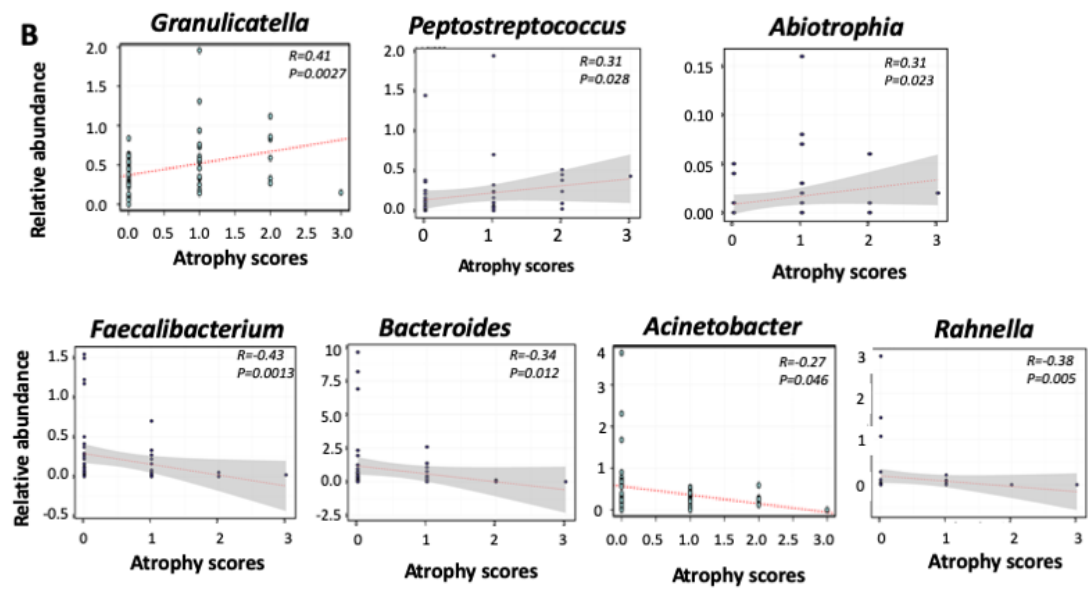

Figure 4 Bacteria associated with atrophy before and after Helicobacter pylori eradication. (A) Linear discriminant analysis (LDA) with effect size (Lefse) showing bacterial genera enriched in patients with atrophy before H. pylori eradication. (B) Spearman correlation of bacterial genera with atrophy scores after H. pylori eradication. (C) LDA with effect size (Lefse) showing bacterial genera enriched or depleted in patients with emerged atrophy 1 year after $H$. pylori eradication. 
A

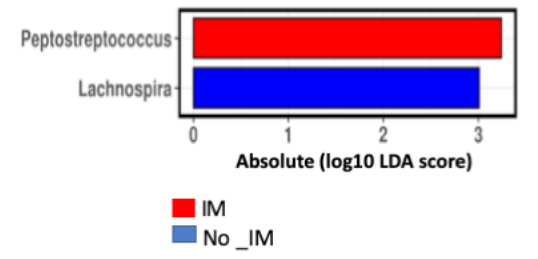

B



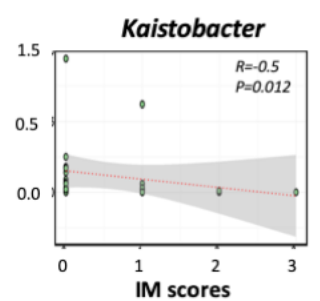
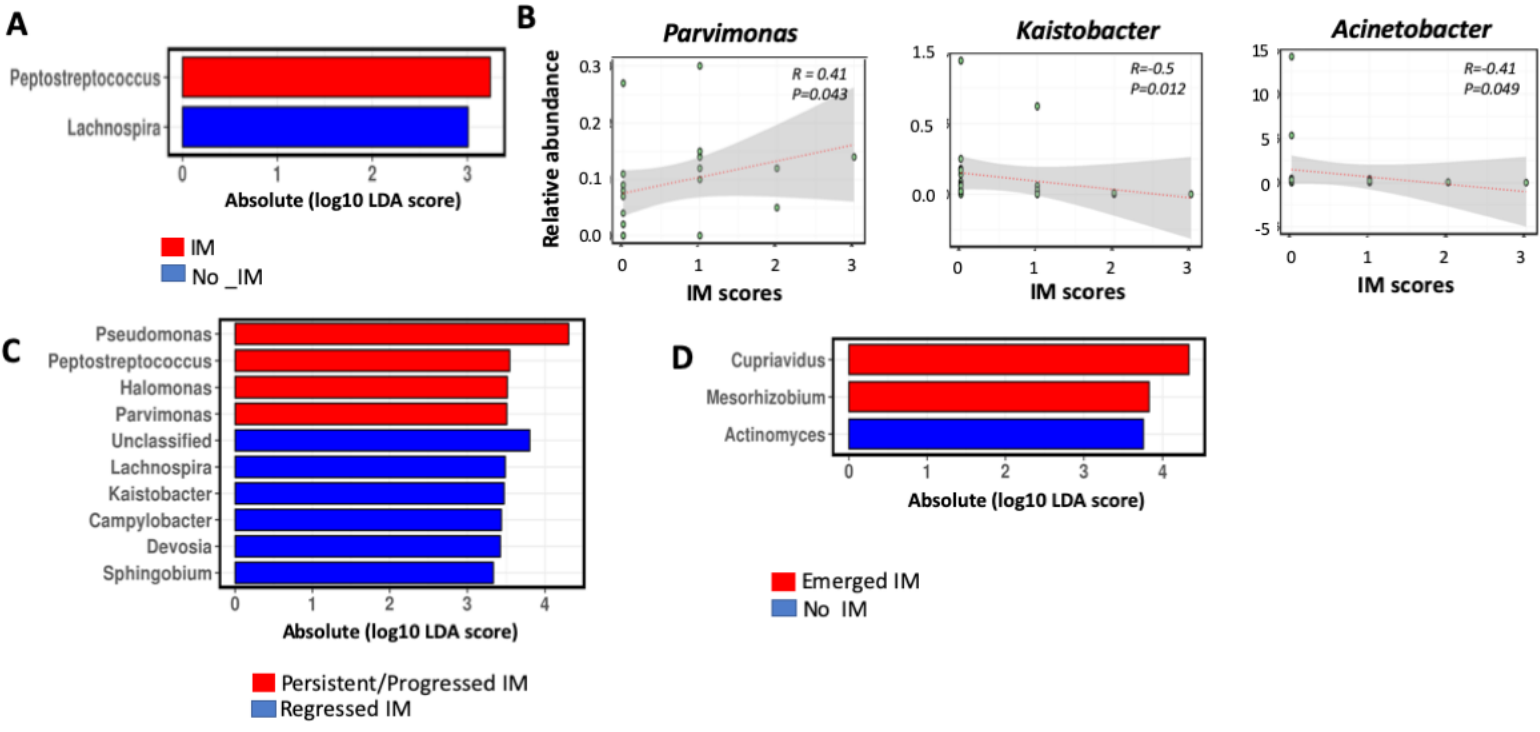

D Cupriavidus

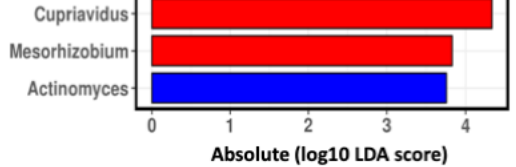

Emerged IM

No IM

Figure 5 Bacteria associated with intestinal metaplasia (IM) before and after Helicobacter pylori eradication. (A) Linear discriminant analysis (LDA) with effect size (Lefse) showing bacterial genera enriched or depleted in patients with IM before H. pylori eradication. (B) Spearman correlation of bacterial genera with IM scores after H. pylori eradication. (C) Linear discriminant analysis (LDA) with effect size (Lefse) showing bacterial genera enriched or depleted in patients with persistent IM or (C) emerged IM 1 year after H. pylori eradication.

\section{Non-H. pylori bacteria associated with intestinal metaplasia}

We further investigated the association of non H. pylori microbes with IM. We found the enrichment of Peptostreptococcus and depletion of Lachnospira in patients with IM before OAC treatment (figure 5A). IM showed very little changes after receiving placebo or OAC. ${ }^{3}$ Correlation analysis determined that Parvimonas $(\mathrm{R}=0.41, \mathrm{p}=0.043)$ was positively correlated with IM scores while Kaistobacter $(\mathrm{R}=-0.5, \mathrm{p}=0.012)$ and Acinetobacter $(\mathrm{R}=-0.41, \mathrm{p}=0.049)$ were negatively correlated in patients with IM prior to $H$. pylori eradication (figure $5 \mathrm{~B}$ ). Among subjects with IM at baseline, Pseudomonas, Peptostreptococcus, Halomonas and Parvimonas were enriched in those with progressed or persisted IM, while Lachnospira, Kaistobacter, Campylobacter, Devosia and Sphingobium were enriched in subjects with regressed IM (figure 5C). Among subjects with no IM at baseline, Mesorhizobium and Cupriavidus were enriched while Actinomyces was depleted in those with emerged IM following $\mathrm{H}$. pylori eradication (figure 5D). We observed that Peptostreptococcus was consistently positively associated with IM before and after OAC treatment.

\section{Functional features of gastric microbiota associated with inflammation, atrophy and IM after $H$. pylori eradication}

The contribution of gut microbiota to pathological conditions are mediated by their functional capabilities. We used PICRUSt to infer the metagenome functional content based on the microbial community profiles obtained from 16S rRNA gene sequences. We found that the inflammation-associated gastric microbiota was enriched in oxidative phosphorylation, methane metabolism, chaperones and folding catalysis, energy metabolism, porphyrin and chlorophyll metabolism, as well as lipid biosynthesis functions, in the absence of $H$. pylori (figure 6A). This observation suggests an increase in energy generation and stress adaptation in the inflammation-associated gastric microbiota. Indeed, stress adaptation of the inflammation-associated microbiota was supported by the enrichment of K03088 which encodes bacterial extracytoplasmic stress response protein RNA polymerase sigma-70 factor. ${ }^{16}$ Other KEGG ontologies such as K09687, K09686, K02005 and K03413 encoding multidrugresistant proteins which are bacterial virulent factors were enriched in inflammation-associated predicted metagenome. Several protein transport encoding genes were reduced in inflammation-associated gastric microbiota (figure 6B).

We also observed a reduction in ribosome biogenesis, linoleic acid metabolism and adipocyte signalling pathways (figure 6C) encoded by predicted genes K01897;long chain acyl coA synthetase, K00528;ferredoxin-NADP+reductase, K03786;3-dehydroquinate dehydratase, K07183;response regulator NasT, K00937; polysphosphate kinase, K00831;phosphoserine aminotransferase, K00818; acetylornithine aminotransferase, K00147;glutamate-5-semialdehyde dehydrogenase and K00031;isocitrate dehydrogenase in aggravated, persistent or emerged atrophy-associated microbiota compared with regressed atrophy following $H$. pylori eradication (figure 6D).

Amino acid metabolism and inositol phosphate metabolism were enriched while folate biosynthesis and NOD-like receptor signalling decreased in subjects whose IM emerged after $H$. pylori eradication (figure 6E). Several KEGG genes encoding virulence bacterial type IV secretion system known to be important in genetic exchange and delivery of effector molecules to eukaryotic target cells ${ }^{17}$ were found enriched in subjects with emerged IM. These include K03195 (VirB10), K03204 (VirB9), K03199 (VirB4) and K03196 (VirB11) (figure 6F). Decrease of K00147;glutamate-5-semialdehyde dehydrogenase was also associated with emergence of IM as observed with atrophy, suggesting its protective importance against the progression of gastric precancerous lesions.

\section{DISCUSSION}

Infection with $H$. pylori is an important risk factor in gastric cancer development, according to Correa's model. ${ }^{18}$ Nevertheless, the risk of gastric cancer reduces only by less than $50 \%$ 
A

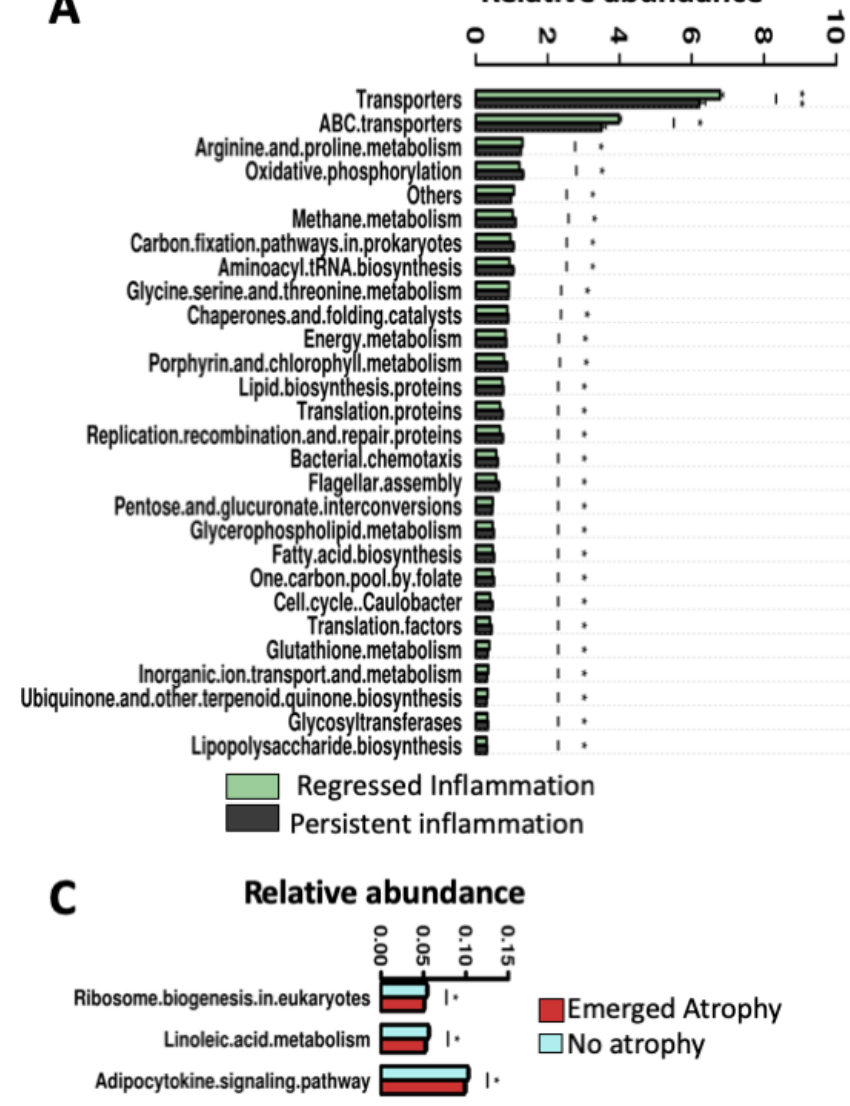

\section{Relative abundance}

(1)

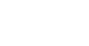

B Relative abundance

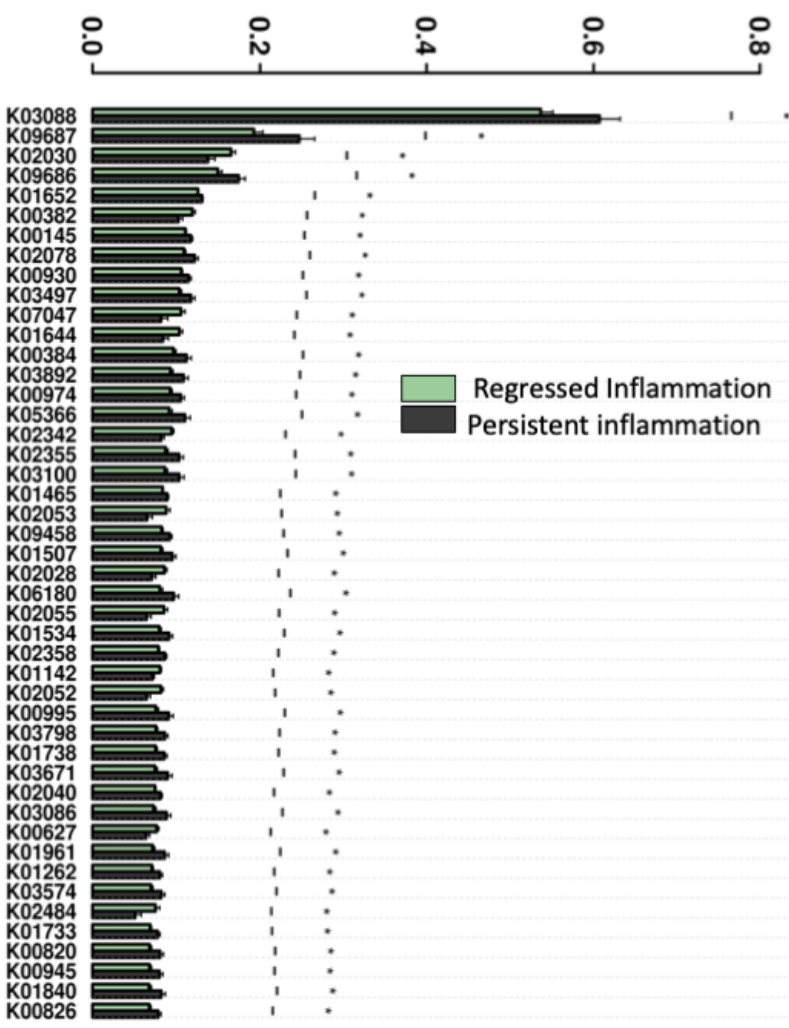

F Relative abundance

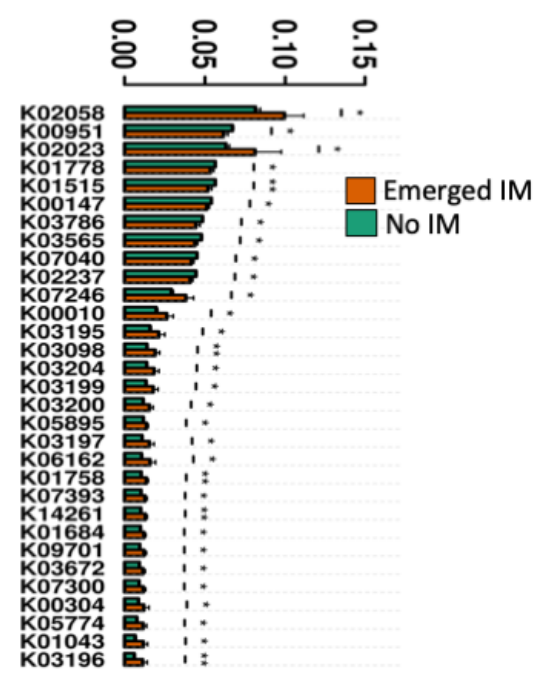

Figure 6 Functional features of gastric microbiota associated with inflammation, atrophy and IM after H. pylori eradication. (A) Predicted microbiota functional changes and (B) corresponding KEGG ontologies in persistent inflammation-associated gastric microbiota. (C) Predicted microbiota functional changes and (D) corresponding KEGG ontologies in GA-associated gastric microbiota. (E) Predicted microbiota functional changes and (F) corresponding KEGG ontologies in persistent IM-associated gastric microbiota. GA, gastric atrophy; IM, intestinal metaplasia; KEGG, Kyoto Encyclopedia of Genes and Genomes.

after $H$. pylori eradication therapy. ${ }^{19}$ As earlier shown by us and others, ${ }^{3}{ }^{19} \mathrm{H}$. pylori eradication therapy alone does not preclude the development of precancerous gastric lesions. We therefore carried out this retrospective study and identified gastric microbes and associated functions that are associated with inflammation, atrophy and IM 1 year after $H$. pylori therapy.

Combined OAC treatment therapy for 1 week effectively eradicated $H$. pylori, ${ }^{3}$ leading to significantly increased bacterial diversity after 1 year. In addition to increased diversity, we observed a significant change in bacteria co-occurrence and a distinct cluster of oral microbes in the absence of $H$. pylori. Haemophilus, Neisseria and Actinobacillus were observed to be significantly reduced after OAC treatment. Coinfection of $H$. pylori and N. subflava has been reported to be associated with lymphoid follicle formation in human stomach. ${ }^{20} \mathrm{~N}$. subflava induced the production of interleukin 8 in gastric epithelial 
cell line and may accelerate the development of gastric lesion in hypochlorhydric stomach. ${ }^{21}$ The depletion of Haemophilus, Neisseria and Actinobacillus as observed in this study may be associated with the consequent reduction in inflammation in the OAC-treated group.

We identified high abundance of pathogenic microbes in subjects with persistent inflammation following $\mathrm{H}$. pylori eradication. The enriched bacteria in subjects with inflammation included Acinetobacter lwoffii, S. anginosus, Ralstonia, Erwinia and Prevotella. A previous study carried out in a mouse model showed that infection with A. lwoffii induced gastrin production, proliferation of gastric $G$ cells and parietal cell and similar gastric inflammation as $\mathrm{H}$. pylori infection, corroborating our observation in this study. ${ }^{8}$ Moreover, Streptococcus was isolated more frequently from the gastric juice of gastritis compared with healthy stomachs. ${ }^{22}$ We and others have shown the increased abundance of Prevotella and S. anginosus in the mucosa of patients with gastric and oesophageal cancer. ${ }^{1423}{ }^{24}$ In particular, S. anginosus is a human pathogen whose oral presence trended with higher risk of gastric cancer. ${ }^{25}$ Ralstonia is a grampositive bacterium with increasing incidence in respiratory infections. ${ }^{26-28}$ It was enriched in the gastric mucosa of patients with gastric cancer before subtotal gastrectomy. ${ }^{29}$ Taken together, the enrichment of these bacteria in subjects with inflammation in this study highlights their potential pathogenic role in promoting gastric inflammation.

As in other gut diseases such as IBD, colitis and colorectal cancer, ${ }^{30} 31$ we found depletion of potentially gut-protective bacteria Roseburia and Sphingomonas in patients with inflammation. Sphingomonas is an aerobic and metabolically versatile bacterial genus that was characterised as being depleted in breast cancer tissues compared with healthy breast tissues. ${ }^{32}$ Roseburia is associated with the formation of butyrate, a short-chain fatty acid reportedly protective against gut neoplasia. ${ }^{33}$ It is important to note that short-chain fatty acids are also produced and absorbed in the stomach ${ }^{34}$ and might be playing protective roles as described in the intestine. ${ }^{35}$ The potential role of probiotics in alleviating gastric inflammation after $H$. pylori eradication has been previously demonstrated, ${ }^{367}$ further supporting our findings in this study and suggest that the prescription of probiotics to patients receiving $H$. pylori eradication therapy may prevent gastric inflammation triggered by other gastric microbes.

The development of gastric atrophy and IM leads to loss of acid-secreting parietal cell. ${ }^{38}$ This may favour the proliferation of gastric microbes or colonisation of the stomach by oral microbes. ${ }^{39}$ We recognised that the emergence of atrophy or IM was associated with increased abundance of Granulicatella, Actinomyces, Rothia, Peptostreptococcus, Streptococcus, Abiotrophia and Parvimonas, in the absence of H. pylori. These are predominantly oral microbes and form a distinct cluster in the microbial ecology of the OAC-treated patients. Their co-occurrence may be important to their neoplasia-promoting effect and subsequent development of gastric cancer as we previously reported..$^{26}$ Granulicatella is gram-positive facultative anaerobe linked to root canal infection and was isolated from the peritoneal fluid of a patient with gastric cancer, ${ }^{40}{ }^{41}$ while Abiotrophia was highlighted as a potential risk element for gastric cancer, ${ }^{25}$ further supporting their potential involvement in development of gastric atrophy. Mesorhizobium, a nitrogen-fixing bacterium, which was characterised as being able to invade human cells, ${ }^{42}$ was enriched in patients whose IM progressed within 1 year following $\mathrm{H}$. pylori eradication. Bacteria producing carcinogenic nitroso compounds through the conversion of nitrates are thought to be important drivers of gastric neoplasia. ${ }^{43}$
Interestingly, Faecalibacterium, Kaistobacter and Rabnella were depleted in subjects whose atrophy emerged following $H$. pylori eradication. Anticarcinogenic role has been demonstrated for F. prausnitzii in GI diseases including IBD, colitis-associated colorectal cancer and hepatocellular carcinoma. ${ }^{44}{ }^{45}$ We identify its potential protective role in gastric neoplasia for the first time in this study. Notably, we observed that different sets of bacteria are associated with atrophy and IM, before and after $H$. pylori eradication. The significance of this is that the presence of $H$. pylori changes the gastric milieu which might influence the microflora of the stomach. It would be interesting to follow the effects of different microbiota in the process of gastric carcinogenesis in the future.

Moreover, we demonstrated that energy generation and adaptation to stress was upregulated in the gut microbiota of subjects with gastric inflammation. This observation is further supported by the enriched bacterial extracytoplasmic stress response protein RNA polymerase sigma-70 factor ${ }^{16}$ in inflammationassociated gastric microbiota. Our finding is compatible with decline of host antioxidant defenses and consequent physiological and oxidative stress that characterise the stomach during gastric inflammation. ${ }^{46}$ Moreover, folate biosynthesis and Nodlike receptor signalling had significantly higher predicted abundances in the gastric microbiota of subjects with IM after $H$. pylori eradication. Folate biosynthesis by the gut microbiota is a source of the water-soluble tetrahydrofolates which play an essential role in human metabolism. ${ }^{47}$ Inadequate status of folate has been implicated in increased risk of colon cancer especially if precancerous lesions exist. ${ }^{48}$ This may be applicable to gastric carcinogenesis, given our observation in this study. Optimisation of bacterial production of folate may reduce the risk of IM development. ${ }^{48}$ Nucleotide oligomerisation domain containing proteins (NOD) are conserved pattern recognition receptors that are stimulated by pathogen-associated molecular patterns (PAMPS). NOD-like receptors (NLR) can regulate innate and adaptive immune response and may participate in carcinogenesis by regulating cell apoptosis. ${ }^{49}$ The observed reduction in NLR signalling by IM-associated gut microbiota may represent a means through which immune response to PAMPS are dampened, consequently promoting the development of gastric lesions. Several predicted genes encoding virulence bacterial type IV secretion system, namely, VirB10, VirB9, VirB4 and VirB11, known to be important in genetic exchange and delivery of effector molecules to eukaryotic target cells ${ }^{17}$ were found enriched in subjects with emerged IM. These data suggest that the gastric microbes reduce folate biosynthesis, dampen host immunity and deliver effector molecules into host cells in promoting IM development.

In conclusion, this study identified gastric pathogenic microbes that are associated with persistent inflammation (A. lwoffii, S. anginosus and Ralstonia) and atrophy/IM (Granulicatella, Actinomyces, Rothia, Peptostreptococcus, Streptococcus, Abiotrophia and Parvimonas) in patients 1 year following $H$. pylori eradication, which are predominantly oral microbes and form a distinct cluster in the microbial ecology in the absence of $H$. pylori. In keeping with the pathogenic ecology, functional pathways including amino acid metabolism and inositol phosphate metabolism were enriched while folate biosynthesis and NOD-like receptor signalling decreased in atrophy/IM-associated gastric microbiota. Thus, our longitudinal study highlights the potential contribution of gastric microbes in the development and perpetuation of precancerous gastric lesions in the absence of $H$. pylori, which may serve as therapeutic targets for the prevention of gastric carcinogenesis. 
Contributors JJYS designed, supervised the study, performed the clinical trial, collected the tissue samples and edited the manuscript. OOC performed bioinformatics analyses and drafted the manuscript. EC, CHS, STYL and HCHL performed laboratory experiments. JY supervised the study and wrote the manuscript.

Funding This project was supported by National Key R\&D Program of China (No. 2018YFC1312100/2018YFC1312102), National Key R\&D Program of China (2017YFE0190700), Science and Technology Program Grant Shenzhen (JCYJ20170413161534162), Vice-Chancellor's Discretionary Fund CUHK, Shenzhen Virtual University Park Support Scheme to CUHK Shenzhen Research Institute.

Competing interests None declared.

Patient consent for publication Obtained.

Ethics approval This study was approved by the Medical Ethics Committee of the Chinese University of Hong Kong.

Provenance and peer review Not commissioned; externally peer reviewed.

Data availability statement All data relevant to the study are included in the article or uploaded as online supplementary information.

Open access This is an open access article distributed in accordance with the Creative Commons Attribution Non Commercial (CC BY-NC 4.0) license, which permits others to distribute, remix, adapt, build upon this work non-commercially, and license their derivative works on different terms, provided the original work is properly cited, appropriate credit is given, any changes made indicated, and the use is non-commercial. See: http://creativecommons.org/licenses/by-nc/4.0/.

\section{ORCID iD}

Jun Yu http://orcid.org/0000-0001-9239-2416

\section{REFERENCES}

1 Correa P. Helicobacter pylori and gastric carcinogenesis. Am J Surg Pathol 1995; 19:S37-43.

2 Díaz P, Valenzuela Valderrama M, Bravo J, et al. Helicobacter pylori and gastric cancer: adaptive cellular mechanisms involved in disease progression. Front Microbiol 2018;9:5.

3 Sung JJY, Lin S-R, Ching JYL, et al. Atrophy and intestinal metaplasia one year after cure of H. pylori infection: a prospective, randomized study. Gastroenterology 2000;119:7-14.

4 Doorakkers E, Lagergren J, Engstrand L, et al. Helicobacter pylori eradication treatment and the risk of gastric adenocarcinoma in a Western population. Gut 2018:67:2092-6.

5 Hooi JKY, Lai WY, Ng WK, et al. Global prevalence of Helicobacter pylori infection: systematic review and meta-analysis. Gastroenterology 2017;153:420-9.

6 Zavros Y, Rieder G, Ferguson A, et al. Gastritis and hypergastrinemia due to Acinetobacter Iwoffii in mice. Infect Immun 2002;70:2630-9.

7 Lofgren JL, Whary MT, Ge Z, et al. Lack of commensal flora in Helicobacter pyloriinfected INS-GAS mice reduces gastritis and delays intraepithelial neoplasia. Gastroenterology 2011;140:210-20.

8 Mommersteeg MC, Yu J, Peppelenbosch MP, et al. Genetic host factors in Helicobacter pylori-induced carcinogenesis: emerging new paradigms. Biochim Biophys Acta Rev Cancer 1869:2018:42-52.

9 Schloss PD, Westcott SL, Ryabin T, et al. Introducing mothur: open-source, platformindependent, community-supported software for describing and comparing microbial communities. Appl Environ Microbiol 2009;75:7537-41.

10 Pruesse E, Peplies J, Glöckner FO. Sina: accurate high-throughput multiple sequence alignment of ribosomal RNA genes. Bioinformatics 2012;28:1823-9.

11 Edgar RC, Haas BJ, Clemente JC, et al. UCHIME improves sensitivity and speed of chimera detection. Bioinformatics 2011:27:2194-200.

12 Langille MGI, Zaneveld J, Caporaso JG, et al. Predictive functional profiling of microbial communities using 165 rRNA marker gene sequences. Nat Biotechnol 2013:31:814-21.

13 Segata N, Izard J, Waldron L, et al. Metagenomic biomarker discovery and explanation. Genome Biol 2011;12:R60.

14 Coker OO, Dai Z, Nie Y, et al. Mucosal microbiome dysbiosis in gastric carcinogenesis. Gut 2018;67:1024-32.

$15 \mathrm{Kim} \mathrm{J,} \mathrm{Kim} \mathrm{N}$, Jo HJ, et al. An appropriate cutoff value for determining the colonization of Helicobacter pylori by the pyrosequencing method: comparison with conventional methods. Helicobacter 2015;20:370-80.

16 Helmann JD. The extracytoplasmic function (ECF) sigma factors. Adv Microb Physiol 2002;46:47-110.

17 Voth DE, Broederdorf LJ, Graham JG. Bacterial type IV secretion systems: versatile virulence machines. Future Microbiol 2012;7:241-57.

18 Correa P, Piazuelo MB, Camargo MC. The future of gastric cancer prevention. Gastric Cancer 2004:7:9-16.

19 Lee Y-C, Chiang T-H, Chou C-K, et al. Association between Helicobacter pylori eradication and gastric cancer incidence: a systematic review and meta-analysis. Gastroenterology 2016;150:1113-24.
20 Nakamura M, Matsui H, Serizawa H, et al. Coinfection of Helicobacter pylori and Neisseria subflava is closely associated with lymph follicle formation in human stomach. Aliment Pharmacol Ther 2006;24:207-13.

21 Miyata N, Hayashi Y, Hayashi S, et al. Lipopolysaccharides from non-Helicobacter pylor gastric bacteria potently stimulate interleukin-8 production in gastric epithelial cells. Clin Trans/ Gastroenterol 2019;10:e00024.

22 Liu JZ, Xue Y, Zhou LY. Detection of gastritis-associated pathogens by culturing of gastric juice and mucosa. Int I Clin Exp Patho 2018;11.

23 Narikiyo M, Tanabe C, Yamada Y, et al. Frequent and preferential infection of Treponema denticola, Streptococcus mitis, and Streptococcus anginosus in esophageal cancers. Cancer Sci 2004;95:569-74.

24 Shiga K, Tateda M, Saijo S, et al. Presence of Streptococcus infection in extraoropharyngeal head and neck squamous cell carcinoma and its implication in carcinogenesis. Oncol Rep 2001;8:245-8.

$25 \mathrm{Wu}$ J, Xu S, Xiang C, et al. Tongue coating microbiota community and risk effect on gastric cancer. J Cancer 2018;9:4039-48.

26 Block C, Ergaz-Shaltiel Z, Valinsky L, et al. Deja vu: Ralstonia mannitolilytica infection associated with a humidifying respiratory therapy device, Israel, June to July 2011. Euro Surveill 2013;18.

27 Kendirli T, Ciftçi E, İnce E, et al. Ralstonia pickettii outbreak associated with contaminated distilled water used for respiratory care in a paediatric intensive care unit. J Hosp Infect 2004;56:77-8.

28 Coenye T, Vandamme P, LiPuma JJ. Ralstonia respiraculi sp. nov., isolated from the respiratory tract of cystic fibrosis patients. Int J Syst Evol Microbiol 2003;53:1339-42.

29 Tseng C-H, Lin J-T, Ho HJ, et al. Gastric microbiota and predicted gene functions are altered after subtotal gastrectomy in patients with gastric cancer. Sci Rep 2016:6:20701

30 Hibberd AA, Lyra A, Ouwehand AC, et al. Intestinal microbiota is altered in patients with colon cancer and modified by probiotic intervention. BMJ Open Gastroenterol 2017:4:e000145

31 Machiels K, Joossens M, Sabino J, et al. A decrease of the butyrate-producing species Roseburia hominis and Faecalibacterium prausnitzii defines dysbiosis in patients with ulcerative colitis. Gut 2014;63:1275-83.

32 Xuan C, Shamonki JM, Chung A, et al. Microbial dysbiosis is associated with human breast cancer. PLoS One 2014;9:e83744.

33 Rivière A, Selak M, Lantin D, et al. Bifidobacteria and butyrate-producing colon bacteria: importance and strategies for their stimulation in the human gut. Front Microbiol 2016:7.

34 Saunders DR. Absorption of short-chain fatty acids in human stomach and rectum. Nutr Res 1991;11:841-7.

35 Chambers ES, Preston T, Frost G, et al. Role of gut microbiota-generated short-chain fatty acids in metabolic and cardiovascular health. Curr Nutr Rep 2018;7:198-206.

36 Francavilla R, Polimeno L, Demichina A, et al. Lactobacillus reuteri strain combination in Helicobacter pylori infection: a randomized, double-blind, placebo-controlled study. J Clin Gastroenterol 2014:48:407-13.

37 Hauser G, Salkic N, Vukelic K, et al. Probiotics for standard triple Helicobacter pylori eradication a randomized, double-blind, placebo-controlled trial. Medicine 2015;94.

38 Michigami $\mathrm{Y}$, Watari J, Ito $\mathrm{C}$, et al. Long-term effects of $\mathrm{H}$. pylori eradication on epigenetic alterations related to gastric carcinogenesis. Sci Rep 2018;8:14369.

39 Shichijo S, Hirata Y. Characteristics and predictors of gastric cancer after Helicobacter pylori eradication. WJG 2018;24:2163-72.

40 Koh YR, Yi J, Kim HH, et al. Discrepant satellitism for identification of Granulicatella adiacens isolates. Ann Lab Med 2014;34:174-6.

41 Hsiao WWL, Li KL, Liu Z, et al. Microbial transformation from normal oral microbiota to acute endodontic infections. BMC Genomics 2012;13:345

42 SC L, Li B, Hung GC, et al. Isolation and characterization of two novel bacteria Afipia cberi and Mesorhizobium hominis from blood of a patient afflicted with fatal pulmonary illness. PLoS One 2013;8:e82673.

43 Smith MGet al. Cellular and molecular aspects of gastric cancer. WJG 2006;12:2979-90.

44 Zhou L, Zhang M, Wang Y, et al. Faecalibacterium prausnitzii produces butyrate to maintain Th17/Treg balance and to ameliorate colorectal colitis by inhibiting histone deacetylase 1. Inflamm Bowel Dis 2018. [Epub ahead of print: 265 2018].

45 Lopez-Siles M, Martinez-Medina M, Abellà C, et al. Mucosa-associated Faecalibacterium prausnitzii phylotype richness is reduced in patients with inflammatory bowel disease. Appl Environ Microbiol 2015;81:7582-92.

46 Suzuki H, Matsuzaki J, Hibi T. Ghrelin and oxidative stress in gastrointestinal tract. J Clin Biochem Nutr 2010:48:122-5.

47 Magnúsdóttir S, Ravcheev D, de Crécy-Lagard V, et al. Systematic genome assessment of B-vitamin biosynthesis suggests co-operation among gut microbes. Front Genet 2015;6:148.

48 Mason JB, Tang SY. Folate status and colorectal cancer risk: a 2016 update. Mol Aspects Med 2017;53:73-9.

49 Inohara N, Nuñez G. The NOD: a signaling module that regulates apoptosis and host defense against pathogens. Oncogene 2001;20:6473-81. 